\title{
Claude's Syndrome without Ptosis Caused by a Midbrain Infarction
}

\author{
Hiromasa Tsuda, Tomoko Fujita, Kaori Maruyama and Masaki Ishihara
}

\begin{abstract}
An 80-year-old man with angina pectoris abruptly developed Claude's syndrome, which consisted of leftsided partial oculomotor nerve palsy without ptosis and right-sided hemiataxia. There were no other neurological abnormalities. Cranial magnetic resonance imaging indicated an infarction of the left inferior paramedian mesencephalic artery, which may have involved the most caudal portion of the oculomotor fascicules. With anti-platelet therapy, the patient became asymptomatic within 10 days. The oculomotor fascicular arrangement in humans remains unclear. Our case suggests that in the oculomotor fascicles, the fibers to the levator palpebrae superioris may be located more in the rostral region than previously hypothesized.
\end{abstract}

Key words: hemiataxia, inferior paramedian mesencephalic artery, oculomotor fascicules, oculomotor nerve, ophthalmoplegia

(Intern Med 54: 1799-1801, 2015)

(DOI: 10.2169/internalmedicine.54.4121)

\section{Introduction}

Claude's syndrome is defined as unilateral oculomotor nerve palsy and contralateral hemiataxia due to the involvement of the caudal midbrain (1-3). In Claude's syndrome, impairment of the oculomotor fascicules (OF) induces ipsilateral oculomotor nerve palsy. Conversely, the oculomotor fascicular arrangement in the midbrain of humans has been a topic of debate (4-7). We herein describe a very rare case of Claude's syndrome without ptosis due to a midbrain infarction. This case suggests new knowledge of the location of the fibers to the levator palpebrae superioris (LP) in the OF.

\section{Case Report}

An 80-year-old Japanese man complained of sudden onset of diplopia in December 2013 and was admitted to our hospital's neurology ward. The patient received aspirin $(81 \mathrm{mg} /$ day) and isosorbide dinitrate (40 mg/day) for angina pectoris. He had no other relevant medical history. On admission, the patient was alert, had a blood pressure of $154 / 86 \mathrm{mmHg}$, and a heart rate of $66 / \mathrm{min}$. The general examination indi- cated no abnormalities. The patient had a corrected visual acuity of 0.8 in both eyes and the funduscopic examination demonstrated cataract in both eyes. The diameter of the pupil was $3 \mathrm{~mm}$ bilaterally in an illuminated room (Fig. 1). Pupil responses to close as well as direct and indirect light responses were prompt in both eyes. There was no relative afferent pupillary defect. Because the palpebral aperture measured $8.5 \mathrm{~mm}$ in both eyes, the LP was spared. Leftsided palsies of the inferior rectus muscle (IR), medial rectus muscle (MR), superior rectus muscle (SR), and inferior oblique muscle (IO) were observed (Fig. 1) which was confirmed by the red glass test. No other cranial nerve impairments other than left-sided partial oculomotor nerve palsy were detected. The patient was right-handed. His hand grasping power was $30 \mathrm{~kg}$ in the right and $25 \mathrm{~kg}$ in the left. In the upper extremities, Barré's sign was negative, and both Barré's sign and Mingazzini's sign was negative in the lower extremities. Manual muscle testing was normal in the neck and extremities. For both sides of the fingers, bend and extension were normal. In the extremities, muscle tonus was normal, and neither muscle atrophy nor fasciculation was observed. The deep tendon reflexes were normal and pathological reflexes were not detected in the extremities. Superficial sensation (touch sensation, pain sensation, temperature 


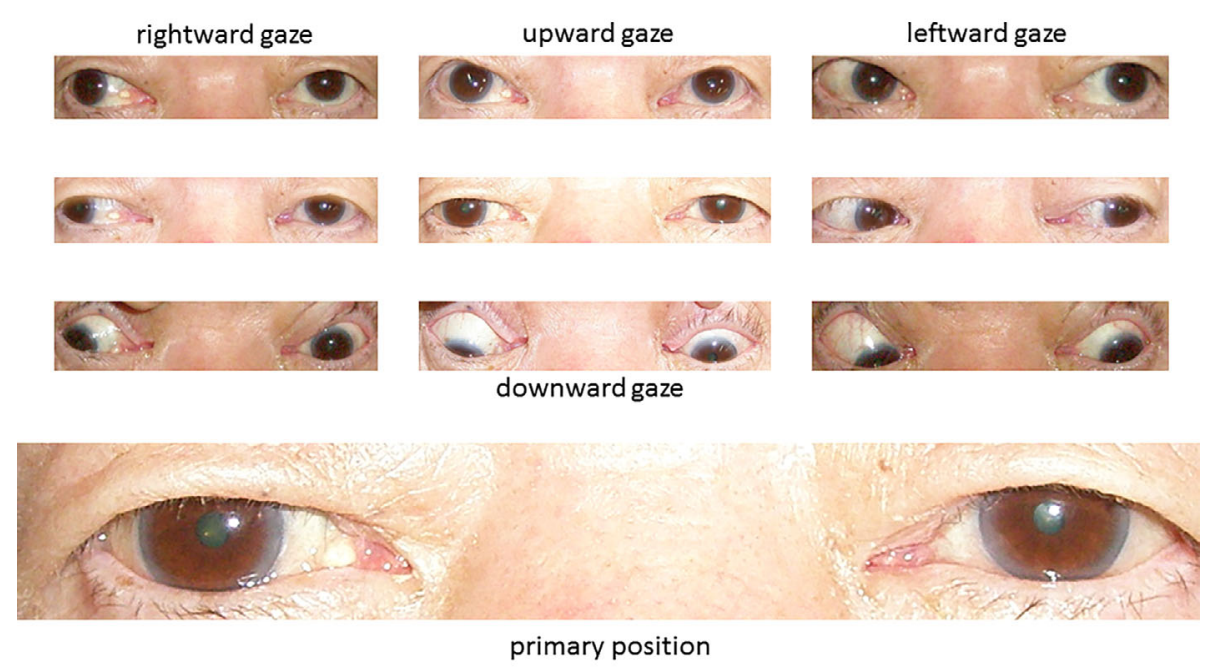

Figure 1. Left-sided paresis of the inferior rectus muscle, medial rectus muscle, superior rectus muscle, and inferior oblique muscle was observed. The pupil diameter was $3 \mathrm{~mm}$ in both eyes in an illuminated room. There was no ptosis in either eye.
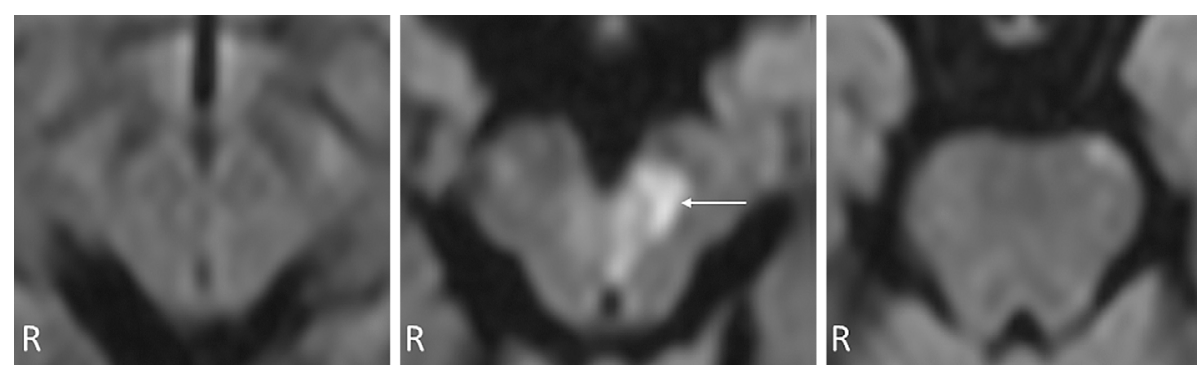

Figure 2. Cranial diffusion-weighted magnetic resonance imaging on axial image demonstrated an infarct lesion in the territory of the inferior paramedian mesencephalic artery on the left side (arrow).

sensation, and topesthesia), deep sensation (joint sensation and vibratory sense), and combined sensation (two-point discrimination, graphesthesia, stereognosis, and double simultaneous stimulation) were all normal. Dysmetria, decomposition of movement, and intension tremor were observed on the right side in the finger-nose test, nose-finger-nose test, and arm stopping test. Furthermore, the hyperpronation test, hand pronation supination test, finger wiggle and foot pat were slower on the right side than on the left side. Rightsided dysmetria were also detected in the heel-knee test and shin-tapping test. Truncal ataxia was not observed, and no abnormalities were detected in the gait test. No other neurological abnormalities were detected. Based on the indications of left-sided partial oculomotor nerve palsy and rightsided hemiataxia, the patient was diagnosed as having Claude's syndrome. The complete blood cell count results and laboratory examination demonstrated no abnormalities. The electrocardiogram findings were normal and the chest roentgenogram demonstrated no abnormalities. Cranial magnetic resonance imaging (MRI) on diffusion-weighted image indicated an infarct lesion in the territory of the inferior paramedian mesencephalic artery on the left side (Fig. 2). However, cranial MRI on coronal image was not performed. Orbital MRI and cranial magnetic resonance angiography demonstrated no abnormalities. With anti-platelet therapy, the patient became asymptomatic within 10 days.

\section{Discussion}

In Claude's syndrome, hemiataxia is hypothesized to be caused by the involvement of the opposite side of the lower red nucleus. However, in 2001, based on the anatomical analysis of six cases of Claude's syndrome, Seo et al. (1) noted that the impairment of the superior cerebellar peduncle led to contralateral hemiataxia. Thereafter, Asakawa et al. (2) and Serrano-Pazo et al. (3) reported further evidence of this hypothesis which was proposed by Seo et al. (1). Consequently, the pathogenesis of hemiataxia in Claude's syndrome remains unclear.

In 1990, Castro et al. (4) speculated that the OF may be a two-dimensional arrangement in which each fiber to the ocular muscles was located somatotopically in the ventral midbrain from lateral to medial as follows: IO, SR, MR, LP, IR, and the sphincter muscle (SP). In 1994, Ksiazek et al. (5) hypothesized a three-dimensional arrangement of the OF (Fig. 3A). Further expanding upon the model proposed by Ksiazek et al., Schwaltz et al. (6) proposed a corrected model of the three-dimensional arrangement of the OF 


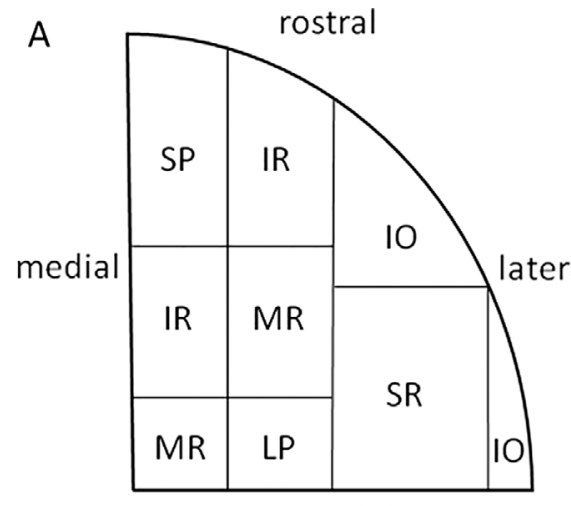

caudal

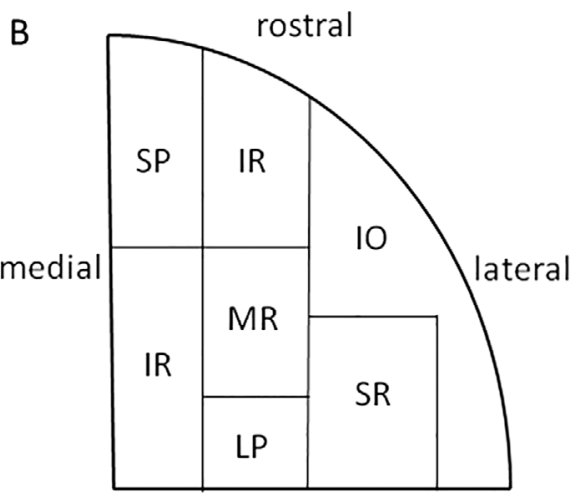

caudal

Figure 3. Proposed models of the three-dimensional arrangement of oculomotor fascicles. A was modified from the model proposed by Ksiazek et al. (5), and B was modified from the model proposed by Schwaltz et al. (6), respectively (IR: inferior rectus muscle, MR: medial rectus muscle, SR: superior rectus muscle, IO: inferior oblique muscle, LP: levator palpebrae superioris, SP: sphincter muscle).

(Fig. 3B). Both models are well-recognized by neurologists and demonstrate that the fibers to the LP may be located in its most caudal portion $(5,6)$.

To the best of our knowledge, there has been only one previously reported case of Claude's syndrome without ptosis due to a midbrain infarction (1). However, the location of the fibers to the LP in the OF was not discussed (1).

The vessels that penetrate the posterior perforated substance and the anterior mesencephalic tegmentum are classified as the paramedian thalamic artery, superior paramedian mesencephalic artery, and inferior paramedian mesencephalic artery (8). Tsuda et al. (7) described two cases of partial fascicular oculomotor paresis with involvement of the LP due to infarction of the paramedian thalamic artery and superior paramedian mesencephalic artery, respectively. In our patient, LP palsy was not observed in infarction of the inferior paramedian mesencephalic artery. Therefore, we speculate that, in the $\mathrm{OF}$, localization of the fibers to the LP is more in the rostral region than previously proposed by the three-dimension models.

The authors state that they have no Conflict of Interest (COI).

\section{References}

1. Seo SW, Heo JH, Lee KY, et al. Localization of Claude's syndrome. Neurology 57: 2304-2307, 2001.

2. Asakawa H, Yanaka K, Nose T. MRI of Claude's syndrome. Neurology 61: 575, 2003.

3. Serrano-Pozo A, Montes-Latorre E, González-Marcos JR, Gil-Peralta A. Cardiac embolism in a Claude's syndrome without involvement of the red nucleus. Eur J Neurol 14: e1-e2, 2007.

4. Castro O, Johnson LN, Mamourian AC. Isolated inferior oblique paresis from brain-stem infarction. Perspective on oculomotor fascicular organization in the ventral midbrain tegmentum. Arch Neurol 47: 235-237, 1990.

5. Ksiazek SM, Slamovits TL, Rosen CE, Burde RM, Parisi F. Fascicular arrangement in partial oculomotor paresis. Am J Ophthalmol 118: 97-103, 1994.

6. Schwartz TH, Lycette CA, Yoon SS, Kargman DE. Clinicoradiographic evidence for oculomotor fascicular anatomy. J Neurol Neurosurg Psychiatry 59: 338, 1995.

7. Tsuda H, Tanaka K. Clinico-anatomical analysis of the fibers to the inferior rectus muscle in the oculomotor fascicles. Intern Med 51: 2031-2034, 2012.

8. Pedroza A, Dujovny M, Ausman JI, et al. Microvascular anatomy of the interpeduncular fossa. J Neurosurg 64: 484-493, 1986.

(C) 2015 The Japanese Society of Internal Medicine http://www.naika.or.jp/imonline/index.html 